This document is confidential and is proprietary to the American Chemical Society and its authors. Do not copy or disclose without written permission. If you have received this item in error, notify the sender and delete all copies.

\title{
Targeted protein degradation through cytosolic delivery of monobody binders using bacterial toxins
}

\begin{tabular}{|r|l|}
\hline Journal: & ACS Chemical Biology \\
\hline Manuscript ID & cb-2019-001133.R1 \\
\hline Manuscript Type: & Article \\
\hline Complete List of Authors: & Schmitted by the \\
& $\begin{array}{l}\text { Schmit, Nadine; Ecole Polytechnique Federale de Lausanne, Swiss } \\
\text { Institute for Experimental Cancer Research (ISREC) } \\
\text { Sciences Katyayanee; Ecole Polytechnique Federale de Lausanne, Life } \\
\text { Hantschel, Oliver; Ecole Polytechnique Federale de Lausanne, Swiss } \\
\text { Institute for Experimental Cancer Research (ISREC) }\end{array}$ \\
\hline
\end{tabular}

\section{SCHOLARONE Manuscripts}




\title{
Targeted protein degradation through cytosolic delivery of monobody binders using
} bacterial toxins

\author{
Nadine Eliane Schmit ${ }^{1,3}$, Katyayanee Neopane ${ }^{1,2,3}$ and Oliver Hantschel ${ }^{1, *}$ \\ ${ }^{1}$ Swiss Institute for Experimental Cancer Research (ISREC), School of Life Sciences, \\ École polytechnique fédérale de Lausanne (EPFL), 1015 Lausanne, Switzerland \\ 2 Present address: Nestlé Institute of Health Sciences, EPFL Innovation Park, 1015 \\ Lausanne, Switzerland \\ ${ }^{3}$ These authors contributed equally to this study \\ *Correspondence and requests for materials should be addressed to O.H. (email: \\ oliver.hantschel@epfl.ch).
}




\begin{abstract}
Monobodies are small engineered binding proteins that, upon expression in cells, can inhibit signaling of cytosolic oncoproteins with outstanding selectivity. Efficacy may be further increased by inducing degradation of monobody targets through fusion to the VHL substrate receptor of the Cullin2-E3 ubiquitin ligase complex. However, potential therapeutic use is currently limited due to the inability of monobody proteins to cross cellular membranes. Here, we use a chimeric bacterial toxin, composed of the Shiga-like toxin B (Stx2B) subunit and the translocation domain of Pseudomonas aeruginosa exotoxin A (ETA-II) for delivery of VHL-monobody protein fusions to target endogenous tyrosine kinases in cancer cells. Depending on the expression of the Stx2B receptor Gb3 on the cell surface, we show that monobodies are taken up by an endocytic route, but are not degraded in lysosomes. Delivery of monobodies fused to a nuclear localization signal resulted in accumulation in the nucleus, thereby indirectly, but unequivocally demonstrating cytosolic delivery. Delivery of VHL fused to monobodies targeting the Lck tyrosine kinase in T-cells resulted in reduced Lck protein levels, which was dependent on expression of Gb3. This led to the inhibition of proximal signaling events downstream of the T-cell receptor complex. This work provides a prime example of the delivery of a stoichiometric protein inhibitor of an endogenous target protein to cells and inducing its degradation without the need of genetic manipulation of target cells. It lays the foundation for further in vivo exploitation of this delivery system.
\end{abstract}




\section{INTRODUCTION}

Targeted cancer therapeutics have improved the survival in several cancer types. Over the past two decades, $\sim 20$ therapeutic antibodies and $\sim 35$ small-molecule enzyme inhibitors targeting key driver oncogenes were developed. ${ }^{1,2}$ Antibodies bind their targets with exquisite selectivity and high affinities, but their application is limited to extracellular targets, as they cannot cross cellular membranes. In contrast, many small molecule inhibitors readily enter cells to inhibit intracellular targets. Engineered binding proteins derived from non-antibody scaffolds (monobodies, DARPins, repebodies, affibodies and others) and mini-immunoglobulin scaffolds (scFvs, Fabs, nanobodies and others) can be readily developed to bind with high affinity and higher selectivity than most small chemical inhibitors to any intracellular target of choice. ${ }^{3,4}$ Their smaller sizes, typically only $10-20 \mathrm{kDa}$, as compared to a full $\mathrm{IgG}$ antibody $(\sim 150 \mathrm{kDa})$ promise better tissue penetration. Still, efficient and tumor-cell selective intracellular protein delivery methods are lacking.

Among the well-studied non-antibody scaffolds are monobodies, synthetic in vitroevolved binders built on the fibronectin type III (FN3) domain. ${ }^{5,6}$ Monobodies are only $\sim 10 \mathrm{kDa}$ in size, lack cysteine residues and can bind their target proteins with low nanomolar affinity. The lack of possible disulfide bridges enables their expression and activity in the reducing environment of the cytoplasm. We and others have extensively used monobodies to target various intracellular oncoproteins, including tyrosine kinases, tyrosine phosphatases, small GTPases and epigenetic regulators. ${ }^{7-12}$ Upon cytosolic expression by plasmid transfection or retro-/lenti-viral gene transfer, monobodies selectively inhibited target-dependent signaling events. In this study, we employ 
monobodies Mb(Lck_1/3) and AS25 that inhibit signaling of the Lck and Bcr-Abl tyrosine kinases, respectively, by targeting its $\mathrm{SH} 2$ domains. 9,10

Targeted protein degradation can lead to a more sustained reduction of signaling, as compared to a small molecule inhibitor alone, as the degradation of the protein-ofinterest also eliminates its scaffolding functions. ${ }^{13,14,15}$ Proteolysis-targeting chimeras (PROTACs) are chemical probes of a protein-of-interest conjugated to a ligand that hijacks either the cereblon or the Cullin2 E3 ligase complex. In contrast to the PROTAC approaches, which require selective high affinity molecular probes that are not available for a large number of therapeutic targets ${ }^{15}$, monobodies can be readily developed against virtually any target of choice. Recently, a monobody (or nanobody) was fused to the Von-Hippel-Lindau (VHL) protein, the substrate receptor of the Cullin2/RBX1 E3 ubiquitin ligase complex, which resulted in degradation of its target protein upon expression in cells. ${ }^{16}$ Other approaches include the Trim-Away method ${ }^{17}$, which requires the genetic overexpression of the E3 ubiquitin ligase Trim21 and electroporation of the cells with antibodies against the target protein. However, the therapeutic use of these approaches is limited, as methods to efficiently deliver recombinant binders to cells are lacking.

Several methods for intracellular delivery of various macromolecular cargos have been studied over the past decades, starting with cell penetrating peptides (CPPs) and including liposomal carriers, diverse nanoparticles and bacterial or viral proteins. ${ }^{18}$ In particular, the efficiency of CPP-mediated delivery is highly cargo- and cell typedependent. ${ }^{19}$ Although several clinical trials with CPPs to deliver drugs, therapeutic peptides and siRNAs to cells have been conducted, none of them has resulted in approval 
of a product. ${ }^{20}$ Most protein delivery studies use cytotoxic proteins, fluorescent probes/proteins or enzymes, such as Cas9, CAT or luciferase, as model cargo. However, an extremely low concentration of such cargos reaching the cytosol can lead to a measurable readout of cellular delivery, even if delivery is very inefficient or the majority of the cargo is entrapped in the endocytic pathway. Examples where protein delivery of a synthetic binding protein of an endogenous oncoprotein results in inhibition of a particular cancer pathway are very limited. ${ }^{21}$

Bacterial toxins have naturally evolved to enter the host cells' cytosol and to escape endosomal degradation. Both high cellular uptake and significant cytoplasmic accumulation of heterologous cargo proteins was achieved with different toxins. ${ }^{22}$ Moreover, cell-selectivity is achieved by binding to a specific host cell receptor. Particularly useful are the so-called AB toxins, which are composed of two subunits, A (for activity, encoding cytotoxic effectors) and B (for binding and uptake into the cytosol). ${ }^{23,24}$ We have adapted a chimeric construct which combines the B-subunit of Shiga-like toxin (Stx2B), secreted by certain pathogenic Escherichia coli strains, with domain II (B subunit) of Exotoxin A, secreted by Pseudomonas aeruginosa (ETA-II). Stx2B is pentameric and binds to globotriaosylceramide (Gb3), a glycosphingolipid, which is present on many human cell types and is upregulated in a number of tumors. ${ }^{25-}$ 28 Both Stx2B and ETA-II follow a retrograde trafficking route in the host cell after endocytosis to escape endosomes. Following furin protease cleavage within the ETA-II domain, the C-terminal portion reaches the cytosol via the Golgi apparatus and the endoplasmatic reticulum (ER). (Figure 1a) The Stx2B-ETAII chimera has been developed and successfully used to deliver EGFP, certain enzymes and an ERK2 kinase 
regulator, and has proven to be more stable when fused to cargo proteins than Stx2B alone. ${ }^{29,30}$

Here, we describe and validate the receptor-specific cytoplasmic delivery of monobodyVHL fusion proteins to cancer cells using a chimeric toxin delivery system, resulting in targeted degradation and signaling inhibition. 


\section{RESULTS AND DISCUSSION}

\section{Cellular uptake of Stx2B-ETA-II-cargo fusion proteins}

The lack of efficient protein delivery to the cytoplasm and nucleus of cancer cells is the major bottleneck for the therapeutic use of synthetic binding proteins. Here, we assess the ability of a chimeric Stx2B-ETA-II toxin system to deliver engineered monobody binders into the cytosol of cancer cells. As the efficiency of any protein delivery system is highly cargo dependent ${ }^{19}$, it is unclear if sufficient amounts of functional monobody can be delivered to target an endogenous signaling pathway. We generated constructs for recombinant expression of either GFP (as control) or different monobodies fused to the C-terminus of Stx2B-ETA-II. (abbreviated as 'toxin' in the remaining paper; Figure 1a) In addition, the constructs contain the ER-retention motif KDEL at the C-terminus, enhancing retrograde transport after furin cleavage of the ETA-II domain. We have also generated constructs incorporating a SNAP-tag for efficient and site-specific labeling with fluorescent benzylguanine (BG) substrates before or after delivery. ${ }^{31}$ Alternatively, and to compare delivery efficiency with the bigger SNAP-tagged constructs, variants with a cysteine at the $\mathrm{C}$-terminus of the monobody were generated, allowing for labeling with a maleimide-coupled fluorophores before delivery. The purity and pentameric nature of all recombinant toxin fusion proteins following affinity purification using a Cterminal 6xHis tag was confirmed by size exclusion chromatography. (Figure 1b, 1c, 1d and Supplementary Figure 1)

We first tested the uptake efficiency of the purified toxin-monobody fusion proteins in HeLa cells. The expression of the Stx2B receptor Gb3 on the surface of HeLa cells was confirmed by flow cytometry and the broad distribution of expression levels is in line 
with literature reports. ${ }^{32}$ (Supplementary Figure 2a) Incubation of HeLa cells with a toxin-emGFP fusion protein resulted in a fluorescent signal in the cytoplasm already after 15 minutes of incubation, demonstrating the correct folding of the recombinant toxin proteins and their ability to deliver a fluorescent protein. (Figure 2a) Similarly, when using a fluorescently labeled monobody as a cargo, we observed an increase in the mean fluorescence over time and efficient uptake at low micromolar concentration. (Figure $2 \mathrm{~b}$ and 2c)

\section{Uptake and absence of degradation of toxin-SNAP-monobody proteins}

To distinguish internalized from surface-bound cargo protein, we made use of cellpermeable and -impermeable fluorogenic SNAP-substrates. We first labeled toxinSNAP-monobody protein with the cell-impermeable SNAP substrate BG-Alexa-Fluor647 (BG-647). Upon incubation with HeLa cells, a weak fluorescent signal was observed in the cytoplasm after only 30 minutes of incubation, which increased with incubation time. (Figure 3a) The staining pattern and increase in signal intensity over time are in line with the data shown in figure $2 \mathrm{a}, 2 \mathrm{~b}$ and $2 \mathrm{c}$, suggesting a similar uptake mechanism and efficiency independent of cargo and fluorescent label. Incubation of the cells with unlabeled toxin-SNAP-monobody constructs and subsequent addition of the cellpermeable BG-Silicorhodamine (BG-SiR) after delivery only showed staining inside the cells. In contrast, no staining was observed with the impermeable BG-647 probe, demonstrating the effective internalization of the toxin-monobody proteins and absence of cell-surface-bound protein. (Figure 3b) 
To assess the fate of the monobody proteins within the cell over longer time periods after delivery, we incubated HeLa cells with BG-647-labeled toxin-SNAP-monobody fusion proteins for 1 hour, followed by a washing step and further incubation in growth medium for up to 24 hours. (Figure 3c) The presence of a robust cytoplasmic fluorescent signal even after a 24-hour incubation in growth medium showed that the proteins remain present in the cell with no signs of degradation.

\section{Endocytosis and cytosolic delivery of toxin-monobody fusions}

In order to study the uptake route, HeLa cells were incubated with toxin-monobody fusion proteins and co-localization with a marker for early endosomes, EEA1, was monitored. We observed co-localization of the delivered protein with EEA1-positive vesicles, which increased over time and decreased thereafter. (Figure $4 \mathrm{a}$ and Supplementary Figure 3) We next tested, if the delivered proteins are trafficked to lysosomes, by analyzing their co-localization with the lysosomal marker Lamp1. Minor co-localization between Lamp1 and protein signals was observed at the earliest timepoint of incubation, and further decreased upon prolonged incubation in growth medium. (Figure $4 \mathrm{~b}$ and Supplementary Figure 4) These results show uptake of the delivered proteins via endocytosis and indicate their translocation to a compartment other than the lysosome.Since unequivocal cytosolic localization is difficult to determine by microscopy due to the complexity of endocytic compartments and dynamic membrane interchange, we used an indirect approach by adding a Nuclear Localization Signal (NLS) to the toxin-monobody construct. (Figure 1a) As NLS recognition takes place in the cytosol, increased nuclear accumulation is therefore an indirect measure of the amount of 
protein that has reached the cytosol and can be monitored by co-localization with a DNA marker. Furthermore, this technique allows imaging of live cells. We observed an NLSdependent increase in nuclear localization of toxin-monobody fusion proteins 24 hours after their delivery, demonstrating at least partial cytosolic uptake of toxin-monobody fusion proteins after delivery. (Figure 4c and Supplementary Figure 5)

\section{Gb3-dependent monobody delivery in cancer cells}

To test if the uptake of Lck-targeting monobodies is Gb3-dependent, we used Gb3negative Jurkat T-cells, which we transduced to inducibly express the lactosylceramide4-alpha-galactosyltransferase (A4GALT; Gb3 synthase). (Supplementary Figure 2b) A4GALT catalyzes the transfer of galactose to lactosylceramide to form $\mathrm{Gb3}$. We monitored the uptake of BG-647 labeled toxin-SNAP-ML3 monobody in Jurkat cells by flow cytometry. Induction of A4GALT expression and incubation with toxin-SNAPML3 showed increased fluorescence, demonstrating that protein uptake is Gb3 receptor dependent. (Figure 5a) To confirm that this signal comes from internalized protein and not from surface-bound protein, we first incubated cells with unlabeled protein and subsequently added the cell-impermeable BG-647 SNAP substrate. These cells emitted a greatly reduced fluorescent signal as compared to cells incubated with pre-labeled protein, demonstrating that the fluorescent signal stems from intracellularly delivered proteins and that very little protein remains bound to $\mathrm{Gb3}$ on the cell surface or unspecifically to the membrane. (Figure 5a)

While the Stx2B receptor Gb3 is not expressed on many leukemia cell lines, its inducible expression gave us the opportunity to control receptor-dependent uptake of toxin- 
monobody fusion proteins. In contrast, $\mathrm{Gb} 3$ is expressed in many primary human tissues and $\mathrm{Gb3}$ expression is upregulated in certain tumor cells, such as Burkitt's lymphoma cells, gastric adenocarcinoma, colorectal cancer cells and others ${ }^{25-28}$ and may therefore enable tumor cell-selective delivery of monobodies in vivo. Additionally, and in order to broaden the applicability of this approach to Gb3-negative tumors, Stx2B could be replaced by binders to other receptors, e.g. repebodies binding to $\mathrm{EGFR}^{30}$ or a DARPin targeting EpCAM ${ }^{23}$, thereby exploiting engagement of tumor cell-specific receptors.

\section{Expression of VHL-monobodies result in Lck degradation}

We have previously shown that the expression of monobodies ML1 and ML3 targeting the Lck kinase inhibited $\mathrm{T}$ cell receptor (TCR) signaling. ${ }^{10}$ We reasoned that we could increase the inhibitory efficacy of ML1 and ML3 by degrading Lck using VHLmonobody fusion proteins. Upon inducible expression of VHL-ML1 or VHL-ML3, but not VHL-HA4_YA, a non-binding control monobody $^{7}$ in Jurkat T-cells, Lck protein levels were reduced by $\sim 50 \%$. (Supplementary Figure $6 \mathrm{a}$ and $6 \mathrm{~b}$ ) Furthermore, the phosphorylation of Zap-70, a direct Lck substrate, was substantially reduced in cells expressing VHL-ML1 or VHL-ML3, but not VHL-HA4_YA, both in unstimulated cells and in cells stimulated with an anti-TCR antibody. (Supplementary Figure 6c and 6d) These results demonstrate the utility of VHL-fused monobodies to degrade Lck and enhance inhibition of TCR signaling.

\section{Delivery of VHL-monobodies result in Lck degradation and inhibition}


The targeted degradation of endogenous proteins without the need of genetic manipulation of cells is of great utility for both research purposes and novel therapeutic avenues. We therefore assessed whether the bacterial toxin delivery system could be used to deliver VHL-monobody fusion proteins and to degrade target proteins.

Toxin-VHL-ML3 and toxin-VHL-HA4_YA fusion proteins were recombinantly expressed and purified. (Supplementary Figure 7) Upon incubation of toxin-VHL-ML3, but not toxin-VHL-HA4_YA or toxin-emGFP with Jurkat T cells, we observed a 50\% decrease of the Lck protein level, indicating protein delivery at similar efficiency as with lentiviral expression. (Figure 5b and 5c) Toxin-VHL-ML3 had no effect on Lck levels in cells that did not express Gb3, demonstrating the exquisite receptor-specificity of this approach. (Figure 5b and 5c) Immunoblot analysis readily detected the delivered Histagged proteins. (Figure 5b) Here, an additional smaller band to the full-length proteins was observed in cells incubated with toxin-ML3 and toxin-emGFP, which corresponds to the size of the protein C-terminal to the furin cleavage site. (C-terminal part of ETA-II, monobody/emGFP, 6xHis-tag and KDEL sequence, Figure 1a and 5b, bottom panel) This indicates that the protein is cleaved by furin along the retrograde pathway. No signal for delivered proteins were detected in cells incubated with ML3 alone and in cells not expressing Gb3, further demonstrating specific and receptor dependent uptake. (Figure $5 b$, bottom panel)

Interestingly, the delivered VHL-fusion constructs were detected at lower levels in cells upon delivery (Figure 5b, bottom panel) than the control proteins not bearing the VHL sequence. This could indicate that the uptake is less efficient due to the larger construct size or due to a higher rate of autodegradation. 
To the best of our knowledge, we demonstrate for the first time the cytosolic delivery of a functional protein binder inducing the targeted degradation of an endogenous target.

We also assessed the effects on cell viability after prolonged incubation with chimeric toxin proteins. Although cell viability remained close to $100 \%$ upon delivery of toxinVHL-monobody, it was reduced when incubating cells for more than 24 hours with toxin-monobody proteins lacking VHL. (Supplementary Figure 8) This was observed in different cell lines, with different monobodies, including a non-binding control and was dependent on the Gb3 receptor. (Supplementary Figure 8) Given the uptake mechanism that exploits retrograde transport through the secretory pathways, it is a plausible hypothesis that the accumulation of proteins in the ER may lead to ER-stress through the UPR pathway. ${ }^{33-35}$ The presence of a relatively unstable domain such as VHL might facilitate unfolding by chaperones and translocation to the cytosol, explaining the absence of toxicity upon toxin-VHL-monobody delivery as compared to the mild toxicity of the toxin-monobody proteins lacking VHL.

We finally wanted to assess whether the reduced Lck protein levels upon VHL-ML3 protein delivery also result in inhibition of TCR signaling, as upon genetic expression of VHL-monobodies. We stimulated Jurkat cells with the anti-TCR antibody after incubation with toxin-VHL-monobody proteins and observed reduced phosphorylation of Zap70 upon delivery of VHL-ML3 as compared to VHL-HA4_YA. (Figure 5d and 5e) These results indicate that VHL-monobody fusion proteins can be delivered, resulting in reduction of endogenous target protein levels and inhibition of downstream signaling.

\section{Future outlook}


While the serum stability and plasma half-life of toxin-monobody proteins remain to be tested, the relatively large size of these pentameric proteins is expected to increase plasma half-life, compared to the known rapid clearance of small protein binders. ${ }^{36}$ However, a likely limitation for the in vivo use of the described method might be systemic immunogenicity derived from the toxin proteins. Previously described methods to remove immunogenic epitopes through protein engineering or to encapsulate proteins could be used to shield the delivered recombinant construct. ${ }^{37}$ Alternatively, the amount of delivered proteins could be reduced through localized applications, such as intratumoral injections or topical application.

The modularity of the described toxin delivery system is of great advantage for various chemical biology and therapeutic applications. As shown, VHL, the self-labeling SNAPtag and a nuclear localization signal can be added to the construct while retaining delivery efficiency. Monobodies can be engineered to bind any intracellular protein of choice Even the addition of a tandem monobody - either binding to two different epitopes on the same domain or engaging two different domains of the same protein could be highly beneficial and boost potency of targeting, as previously demonstrated. ${ }^{8,38}$ Combining the delivery of an allosteric monobody, like ML3, which binds to the SH2 domain of Lck, with an ATP-competitive small molecule kinase inhibitor, targeting the ATP binding site of Lck or a downstream kinase, is an emerging and highly attractive concept. A major advantage of such a combination could include reduced resistance development.

In conclusion, we used a chimeric bacterial toxin to achieve cytoplasmic delivery of a functional monobody protein bound to the substrate binding receptor of an E3 ubiquitin 
ligase complex, resulting in targeted degradation and inhibition of a key signaling protein. This versatile approach offers great promise for future therapeutic use and to specifically degrade any protein of interest without the need of genetic manipulation of cells.

\section{METHODS}

\section{Plasmids and reagents}

The cDNA encoding A4GALT was obtained from the Gene Expression Core Facility at EPFL and cloned into the pEM24 vector (modified pCW22 ${ }^{39}$ obtained from E. Meylan, EPFL) using InFusion recombinase (Clontech), for lentiviral transduction and inducible expression. The cDNA encoding VHL was obtained from the laboratory of G. Sapkota (Dundee University, UK) and VHL-monobody constructs were cloned into the pEM24 vector using InFusion cloning. All constructs in the pEM24 vector were transformed in the E. coli strain HB101. The lentiviral expression system vectors pCMV-R8_74 (encoding gag and pol proteins) and pMD2_G (encoding VSV-G envelope) were kind gifts from I. Barde (Trono Lab, EPFL).

A C-terminal cysteine was introduced at the C-terminus of the monobodies by site directed mutagenesis using the Quik-change site directed mutagenesis kit (Stratagene).

The pET21a vector for recombinant expression containing the Stx2B-ETAII construct was obtained from the laboratory of H.-S. Kim (KAIST, Korea), and monobody, SNAPmonobody, VHL-monobody or monobody-NLS constructs were cloned into this vector. All DNA constructs were verified by DNA sequencing, performed by Microsynth. 


\begin{abstract}
Antibodies and reagents
Antibodies against (Lck (\#2657), Zap70 (\#2709), pZap70(Y319) (\#2701) and pZap70(Y493) (\#2704) were purchased from Cell Signaling Technology, the antibody used for Jurkat cell stimulation (T-cell receptor, clone C305 [\#05-919)) was purchased from Millipore, antibodies against beta-Actin (MA1-140) and against the Myc tag (MA121316-D800, directly coupled to DyLight800) were purchased from ThermoFisher. The antibody against penta-His (34610) was purchased from QIAGEN. Anti-mouse IRDye680 (926-32210), Anti-mouse IRDye800 (925-32210) and anti-rabbit IRDye680 (925-68071) antibodies were purchased from LiCOR. Antibodies used for immunofluorescence against EEA1 (BD610547), Lamp1 (BD555798) and anti-Mouse coupled to Fluorescein isothiocyanate (FITC, F0257) were purchased from Sigma. Maleimide coupled to AlexaFluor488 was purchased from ThermoFisher (A10254), fluorogenic SNAP substrates (Benzylguanine-Silicorhodamine and BenzylguanineAlexaFluor647) were a kind gift from K. Johnsson (EPFL). FITC-conjugated anti-CD77 antibody (357103) was purchased from Biolegend.
\end{abstract}

\title{
Protein labeling \\ Toxin-Monobody constructs bearing a C-terminal cysteine were incubated with maleimide coupled to AlexaFluor488 (ThermoFisher) at a 10-fold molar excess over night at $4^{\circ} \mathrm{C}$ with mild shaking in the dark. Proteins bearing a SNAP-tag were incubated with Benzylguanine-AlexaFluor647 at 5-fold molar excess for 2 hours in the dark at room temperature with mild shaking.
}


Labeled protein was purified using a PD SpinTrap G-25 or PD MidiTrap G-25 by following the manufacturer's instructions and using SEC buffer $(25 \mathrm{mM}$ Tris-HCl $\mathrm{pH} 7.5$, $150 \mathrm{mM} \mathrm{NaCl}, 5 \%$ (w/v) glycerol).

For in-cell labeling, cells previously incubated with the SNAP-protein were incubated with $500 \mathrm{nM}$ permeable SNAP substrate (benzylguanine-Silicorhodamine) for 30 minutes, prior to washing with PBS.

\section{Image processing and analysis}

After image acquisition, the images were processed and analyzed in Fiji/ImageJ software using the Common Tools Plugin (BIOP, EPFL). The same settings were applied to all the images from one experiment.

For colocalization analysis, regions of interest (ROIs) describing the cell area were selected from the processed images using the MultiManualSelect tool (BIOP, EPFL). All experiments were done in at least two biological repeats and at least 15-20 images per experimental condition were analyzed with 1-4 cells per image. After selecting ROIs, a threshold algorithm was selected for each channel to distinguish true signal from background noise for each image. Mander's overlap coefficients between the channels for the protein signal and the antibody signal were calculated using the JaCOP tool (BIOP, EPFL), for each individual ROI by averaging over the different z-stacks.

For nuclear localization analysis, an ImageJ script was used to define de Hoechst-stained nucleus as ROI in each cell, measure the signal from the $488 \mathrm{~nm}$ channel in the defined ROI (nucleus) for each z-plane individually and average the values over the different $z-$ planes. 
A detailed description of Methods is available as Supporting Information.

\begin{abstract}
ACKNOWLEDGEMENTS
This work was supported by the European Research Council (Grant ERC-2016-CoG 682311-ONCOINTRABODY) and the National Center of Competence in Research (NCCR) in Chemical Biology. We thank T. Kükenshöner, G. Mann and T. Reichart for their input on the manuscript, S. Koide for critical discussions, R. Guiet and O. Burri (EPFL Bioimaging and Optics Platform) for their help with microscopy image processing and analysis and all members of the Hantschel lab for continuous support and discussions. We thank H.-S. Kim for the bacterial toxin DNA construct and G. Sapkota for VHL DNA constructs.
\end{abstract}

Supporting Information Available: This material is available free of charge via the Internet.

Supplementary Methods

Supplementary Figures 1-9

\title{
FIGURE LEGENDS
}

Figure 1. Expression and purification of recombinant toxin-monobody fusion proteins. (a) Schematic of the constructs with their monomeric and pentameric size in kDa. (b) Size exclusion chromatogram of StxB-ETAII-ML3 as representative for the other purified proteins. (c) Coomassie stained SDS-PAGE gel of StxB-TDP-ML3 with the fractions 
from the Ni-NTA purification ( $\mathrm{L}=$ crude lysate, $\mathrm{FT}=$ flow-through, $\mathrm{W}=$ wash, $\mathrm{E}=\mathrm{elution}$ ) and the main peak of the SEC after concentration. (d) Corresponding immunoblot with an antibody recognizing penta-His.

Figure 2. Toxin-monobody delivery in HeLa cells. (a) HeLa cells were incubated with 1 $\mu \mathrm{M}$ toxin-emGFP and imaged at different time points. (b) HeLa cells were incubated with $1 \mu \mathrm{M}$ AF488-labeled toxin-AS25 ${ }^{9}$ and imaged at different time points. (c) HeLa cells were incubated with $0.1 \mu \mathrm{M}$ or $1 \mu \mathrm{M}$ or $2.5 \mu \mathrm{M}$ toxin-AS25 for 1 h. Live cells were imaged on a confocal microscope. Scale bars correspond to $10 \mu \mathrm{m}$. Image quantification of these experiments are shown in Supplementary Fig. 2

Figure 3. Life-cell imaging of delivery of SNAP-tagged toxin-monobody fusion proteins in HeLa cells. (a) HeLa cells were incubated with toxin-SNAP-AS25 prelabeled with BG-647 for the indicated durations. (b) HeLa cells were incubated with unlabeled toxinSNAP-ML3 for the indicated durations, washed and incubated with either BG-SiR or BG-647 for 30 min. The bottom image shows HeLa cells incubated only with BG-SiR, but no protein. (c) HeLa cells were incubated for $1 \mathrm{~h}$ with BG-647-labeled toxin-SNAPAS25, washed and incubated in medium for $2.5,9$ or 24 hours. Live cells were imaged on a confocal microscope. Scale bars correspond to $10 \mu \mathrm{m}$. Image quantification of these experiments are shown in Supplementary Fig. 2

Figure 4. Subcellular localization of toxin-monobody fusion proteins. (a) Colocalization analysis of BG-647 labeled toxin-SNAP-AS25 with early endosomes in HeLa cells. HeLa cells were incubated with the protein for 10 minutes, washed, incubated in growth medium and fixed after 0,10 or 30 min or $1 \mathrm{~h}$. Early endosomes were stained with an antibody against EEA1 and the Mander's overlap coefficient 2 between the antibody and 
the protein signals is plotted for each cell. (Plots of Mander's 1 versus 2 are shown in supplementary figure S3.) $P$-values were calculated using a Welch two-sample t-test. (b) Colocalization analysis of BG-647 labeled toxin-SNAP-AS25 with lysosomes in HeLa cells. Lysosomes were stained with an antibody against Lamp1 and the Mander's overlap coefficient 2 between the antibody and the protein signals is plotted for each cell. (Plots of Mander's 1 versus 2 are shown in supplementary figure S4.) (c) Uptake of NLStagged toxin-monobody proteins in the nucleus. HeLa cells were incubated with AF-488 labeled toxin-ML3-NLS or toxin-AS25-NLS or toxin-AS25 (without NLS) for 2.5h, washed and incubated in growth medium for the indicated total times. The fluorescence intensity of the $488 \mathrm{~nm}$ signal in the nucleus stained with Hoechst was quantified from confocal microscopy images of live cells. Each dot represents the mean $488 \mathrm{~nm}$ fluorescence in the nucleus of a single cell, normalized to the mean of the control cells incubated with unlabeled toxin-AS25-NLS. $P$-values were calculated using a Welch twosample t-test. Boxplots represent the median value, the first and third quartiles (lower and upper hinges) and the smallest and largest value within 1.5 times the interquartile range (lower and upper whiskers). An independent repeat of the experiment with additional timepoints is shown in Supplementary Fig. 5D.

Figure 5. Delivery of toxin-VHL-ML3 in Jurkat cells. (a) A4GALT-transduced Jurkat cells were treated with doxycycline for $24 \mathrm{~h}$ to induce Gb3 expression (blue, orange and purple lines) or left untreated (red and green lines). Uninduced cells were incubated with BG-647 labeled toxin-SNAP-ML3 for 30 minutes (green line); doxycycline-induced cells were incubated with unlabeled toxin-SNAP-ML3 for 30 minutes, washed and subsequently incubated with BG-647 (orange line); doxycycline-induced cells were 
incubated with BG-647 labeled toxin-SNAP-ML3 for 30 minutes (purple line). All cells were washed and analyzed by flow cytometry. One representative plot is shown out of 2 biological repeats. (b) Expression of Gb3 was induced by addition of doxycycline in Jurkat cells as indicated and cells were incubated for 48 hours with the indicated proteins, washed and lysed. The cell lysate was immunoblotted with antibodies against Lck, Actin and penta-His. (c) Quantification of the Lck immunoblot normalized to Actin and to the control where no protein was added. Each dot represents a biological repeat of the experiment. Toxin-VHL-MbCtrl means that either toxin-VHL-HA4_YA or toxin-VHLAS25 was used as a control ( 2 repeats of each). $P$-values were calculated using a twotailed t-test. $\left({ }^{*} p<0.05,{ }^{* *} \mathrm{p}<0.005\right)$ Error bars indicate the SD of the repeats. (d) Jurkat cells expressing Gb3 (induced with doxycycline) were incubated for 48 hours with the indicated proteins. Cells were stimulated with an anti-TCR antibody for 5 minutes and lysed. Immunoblot analysis of the cell lysate with antibodies against Lck, Actin, phosphorylated Y319 residue of Zap70, total Zap70 and His-tagged proteins are shown from top to bottom. One representative blot is shown from 3 biological repeats. Quantification of the Lck immunoblot normalized to Actin is shown in Supplementary Fig. 7 (e) Quantification of the pY319 Zap70 immunoblot normalized to the loading control (Actin or total Zap70) and to the unstimulated cells incubated with toxin-VHLHA4_YA from 3 biological repeats. P-values were calculated using a ratio paired t-test. $\left({ }^{*} \mathrm{p}<0.05\right)$ Error bars indicate the SD of the repeats. 


\section{REFERENCES}

(1) Carter, P. J., and Lazar, G. A. (2018) Next generation antibody drugs: pursuit of the 'high-hanging fruit'. Nat. Rev. Drug Discov. 17, 197-223.

(2) Wang, Q., Zorn, J. A., and Kuriyan, J. (2014) A structural atlas of kinases inhibited by clinically approved drugs. Meth. Enzymol. 548, 23-67.

(3) Sha, F., Salzman, G., Gupta, A., and Koide, S. (2017) Monobodies and other synthetic binding proteins for expanding protein science. Protein Sci. 26, 910-924.

(4) Hantschel, O. (2017) Monobodies as possible next-generation protein therapeutics - a perspective. Swiss Med Wkly 147, w14545.

(5) Koide, A., Bailey, C. W., Huang, X., and Koide, S. (1998) The fibronectin type III domain as a scaffold for novel binding proteins. J. Mol. Biol. 284, 1141-1151.

(6) Koide, A., Wojcik, J., Gilbreth, R. N., Hoey, R. J., and Koide, S. (2012) Teaching an old scaffold new tricks: monobodies constructed using alternative surfaces of the FN3 scaffold. J. Mol. Biol. 415, 393-405.

(7) Wojcik, J., Hantschel, O., Grebien, F., Kaupe, I., Bennett, K. L., Barkinge, J., Jones, R. B., Koide, A., Superti-Furga, G., and Koide, S. (2010) A potent and highly specific FN3 monobody inhibitor of the Abl SH2 domain. Nat. Struct. Mol. Biol. 17, 519-527.

(8) Sha, F., Gencer, E. B., Georgeon, S., Koide, A., Yasui, N., Koide, S., and Hantschel, O. (2013) Dissection of the BCR-ABL signaling network using highly specific monobody inhibitors to the SHP2 SH2 domains. Proc. Natl. Acad. Sci. U. S. A. 110, $14924-14929$.

(9) Wojcik, J., Lamontanara, A. J., Grabe, G., Koide, A., Akin, L., Gerig, B., Hantschel, O., and Koide, S. (2016) Allosteric Inhibition of Bcr-Abl Kinase by High Affinity Monobody Inhibitors Directed to the Src Homology 2 (SH2)-Kinase Interface. J. Biol. Chem. 291, 8836-8847.

(10) Kükenshöner, T., Schmit, N. E., Bouda, E., Sha, F., Pojer, F., Koide, A., Seeliger, M., Koide, S., and Hantschel, O. (2017) Selective Targeting of SH2 Domain-

Phosphotyrosine Interactions of Src Family Tyrosine Kinases with Monobodies. J. Mol. Biol. 429, 1364-1380.

(11) Spencer-Smith, R., Koide, A., Zhou, Y., Eguchi, R. R., Sha, F., Gajwani, P., Santana, D., Gupta, A., Jacobs, M., Herrero-Garcia, E., Cobbert, J., Lavoie, H., Smith, M., Rajakulendran, T., Dowdell, E., Okur, M. N., Dementieva, I., Sicheri, F., Therrien, M., Hancock, J. F., Ikura, M., Koide, S., and O'Bryan, J. P. (2017) Inhibition of RAS function through targeting an allosteric regulatory site. Nat. Chem. Biol. 13, 62-68. (12) Gupta, A., Xu, J., Lee, S., Tsai, S. T., Zhou, B., Kurosawa, K., Werner, M. S., Koide, A., Ruthenburg, A. J., Dou, Y., and Koide, S. (2018) Facile target validation in an animal model with intracellularly expressed monobodies. Nat. Chem. Biol. 14, 895-900. (13) Winter, G. E., Buckley, D. L., Paulk, J., Roberts, J. M., Souza, A., Dhe-Paganon, S., and Bradner, J. E. (2015) Phthalimide conjugation as a strategy for in vivo target protein degradation. Science 348, 1376-1381.

(14) Lai, A. C., and Crews, C. M. (2017) Induced protein degradation: an emerging drug discovery paradigm. Nat. Rev. Drug Discov. 16, 101-114.

(15) Burslem, G. M., Smith, B. E., Lai, A. C., Jaime-Figueroa, S., McQuaid, D. C., Bondeson, D. P., Toure, M., Dong, H., Qian, Y., Wang, J., Crew, A. P., Hines, J., and 
Crews, C. M. (2018) The Advantages of Targeted Protein Degradation Over Inhibition: An RTK Case Study. Cell Chem. Biol. 25, 67-77.

(16) Fulcher, L. J., Hutchinson, L. D., Macartney, T. J., Turnbull, C., and Sapkota, G. P. (2017) Targeting endogenous proteins for degradation through the affinity-directed protein missile system. Open Biol. 7, 170066.

(17) Clift, D., McEwan, W. A., Labzin, L. I., Konieczny, V., Mogessie, B., James, L. C., and Schuh, M. (2017) A Method for the Acute and Rapid Degradation of Endogenous Proteins. Cell 171, 1692-1706.

(18) Bruce, V. J., and McNaughton, B. R. (2017) Inside Job: Methods for Delivering Proteins to the Interior of Mammalian Cells. Cell Chem. Biol. 24, 924-934.

(19) Conner, S. D., and Schmid, S. L. (2003) Regulated portals of entry into the cell. Nature 422, 37-44.

(20) Koren, E., and Torchilin, V. P. (2012) Cell-penetrating peptides: breaking through to the other side. Trends Mol. Med. 18, 385-393.

(21) Liao, X., Rabideau, A. E., and Pentelute, B. L. (2014) Delivery of Antibody Mimics into Mammalian Cells via Anthrax Toxin Protective Antigen. Chembiochem 15, 24582466.

(22) Guillard, S., Minter, R. R., and Jackson, R. H. (2015) Engineering therapeutic proteins for cell entry: the natural approach. Trends in biotechnology.

(23) Verdurmen, W. P. R., Luginbühl, M., Honegger, A., and Plückthun, A. (2015) Efficient cell-specific uptake of binding proteins into the cytoplasm through engineered modular transport systems. J. Control. Release 200, 13-22.

(24) Beilhartz, G. L., Sugiman-Marangos, S. N., and Melnyk, R. A. (2017) Repurposing bacterial toxins for intracellular delivery of therapeutic proteins. Biochem. Pharmacol. $142,13-20$.

(25) Janssen, K.-P., Vignjevic, D., Boisgard, R., Falguières, T., Bousquet, G., Decaudin, D., Dollé, F., Louvard, D., Tavitian, B., Robine, S., and Johannes, L. (2006) In vivo tumor targeting using a novel intestinal pathogen-based delivery approach. Cancer Res. $66,7230-7236$.

(26) Taga, S., Carlier, K., Mishal, Z., Capoulade, C., Mangeney, M., Lécluse, Y., Coulaud, D., Tétaud, C., Pritchard, L. L., Tursz, T., and Wiels, J. (1997) Intracellular signaling events in CD77-mediated apoptosis of Burkitt's lymphoma cells. Blood 90, 2757-2767.

(27) Geyer, P. E., Maak, M., Nitsche, U., Perl, M., Novotny, A., Slotta-Huspenina, J., Dransart, E., Holtorf, A., Johannes, L., and Janssen, K.-P. (2016) Gastric Adenocarcinomas Express the Glycosphingolipid Gb3/CD77: Targeting of Gastric Cancer Cells with Shiga Toxin B-Subunit. Mol. Cancer Ther. 15, 1008-1017. (28) Kovbasnjuk, O., Mourtazina, R., Baibakov, B., Wang, T., Elowsky, C., Choti, M. A., Kane, A., and Donowitz, M. (2005) The glycosphingolipid globotriaosylceramide in the metastatic transformation of colon cancer. Proc. Natl. Acad. Sci. U. S. A. 102, 1908719092.

(29) Ryou, J.-H., Sohn, Y.-K., Kim, D.-G., Kyeong, H.-H., and Kim, H.-S. (2018) Engineering and cytosolic delivery of a native regulatory protein and its variants for modulation of ERK2 signaling pathway. Biotechnol. Bioeng. 115, 839-849. (30) Ryou, J.-H., Sohn, Y.-K., Hwang, D.-E., Park, W.-Y., Kim, N., Heo, W.-D., Kim, M.-Y., and Kim, H.-S. (2016) Engineering of bacterial exotoxins for highly efficient and 
receptor-specific intracellular delivery of diverse cargos. Biotechnol. Bioeng. 113, 16391646.

(31) Keppler, A., Gendreizig, S., Gronemeyer, T., Pick, H., Vogel, H., and Johnsson, K. (2003) A general method for the covalent labeling of fusion proteins with small molecules in vivo. Nat. Biotechnol. 21, 86-89.

(32) Yamaji, T., and Hanada, K. (2014) Establishment of HeLa cell mutants deficient in sphingolipid-related genes using TALENs. PloS one 9, e88124.

(33) Shen, X., Zhang, K., and Kaufman, R. J. (2004) The unfolded protein response--a stress signaling pathway of the endoplasmic reticulum. J. Chem. Neuroanat. 28, 79-92. (34) Sitia, R., and Braakman, I. (2003) Quality control in the endoplasmic reticulum protein factory. Nature 426, 891-894.

(35) Cudna, R. E., and Dickson, A. J. (2003) Endoplasmic reticulum signaling as a determinant of recombinant protein expression. Biotechnol. Bioeng. 81, 56-65.

(36) Mitchell, T., Chao, G., Sitkoff, D., Lo, F., Monshizadegan, H., Meyers, D., Low, S., Russo, K., DiBella, R., Denhez, F., Gao, M., Myers, J., Duke, G., Witmer, M., Miao, B., Ho, S. P., Khan, J., and Parker, R. A. (2014) Pharmacologic profile of the Adnectin BMS-962476, a small protein biologic alternative to PCSK9 antibodies for low-density lipoprotein lowering. J. Pharmacol. Exp. Ther. 350, 412-424.

(37) Du, J., Jin, J., Yan, M., and Lu, Y. (2012) Synthetic nanocarriers for intracellular protein delivery. Curr. Drug Metab. 13, 82-92.

(38) Grebien, F., Hantschel, O., Wojcik, J., Kaupe, I., Kovacic, B., Wyrzucki, A. M., Gish, G. D., Cerny-Reiterer, S., Koide, A., Beug, H., Pawson, T., Valent, P., Koide, S., and Superti-Furga, G. (2011) Targeting the SH2-kinase interface in Bcr-Abl inhibits leukemogenesis. Cell 147, 306-319.

(39) Meylan, E., Dooley, A. L., Feldser, D. M., Shen, L., Turk, E., Ouyang, C., and Jacks, T. (2009) Requirement for NF-kappaB signalling in a mouse model of lung adenocarcinoma. Nature 462, 104-107. 
a

Monomer Pentamer Nomenclature

Size $(\mathrm{kDa})$ Size $(\mathrm{kDa})$ used in this paper

\begin{tabular}{lll}
\hline 50 & 250 & Toxin-emGFP \\
34 & 170 & Toxin-monobody \\
53 & 265 & Toxin-SNAP-monobody \\
35 & 175 & Toxin-monobody-NLS \\
59 & 295 & Toxin-VHL-monobody
\end{tabular}

Furin

cleavage site

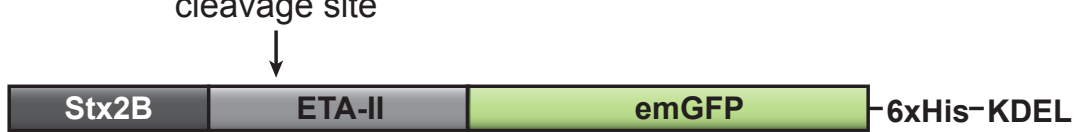

\begin{tabular}{l|l|l|} 
Stx2B & ETA-II & Monobody -6xHis-KDEL
\end{tabular}

\begin{tabular}{|l|l|l|l|}
\hline Stx2B & ETA-II & SNAP & Monobody -6xHis-KDEL
\end{tabular}

\begin{tabular}{l|l|l|l|}
\hline Stx2B & ETA-II & Monobody & NLS -6xHis-KDEL
\end{tabular}

\begin{tabular}{|l|l|l|l|} 
Stx2B & ETA-II & VHL & Monobody -6xHis-KDEL
\end{tabular}

b

Toxin-ML3

C

Toxin-ML3

d

Toxin-ML3

17 L FT W E SEC

8

2070

22

23

23

24

25

26

$\begin{array}{lllll}670 \quad 158 \quad 44 & 17 & 1.35 & k D a\end{array}$ $00 \mathrm{kD}$

$70 \mathrm{kD}$

$55 \mathrm{kD}$

$40 \mathrm{kD}$

$35 \mathrm{kD}$

$25 \mathrm{kD}$
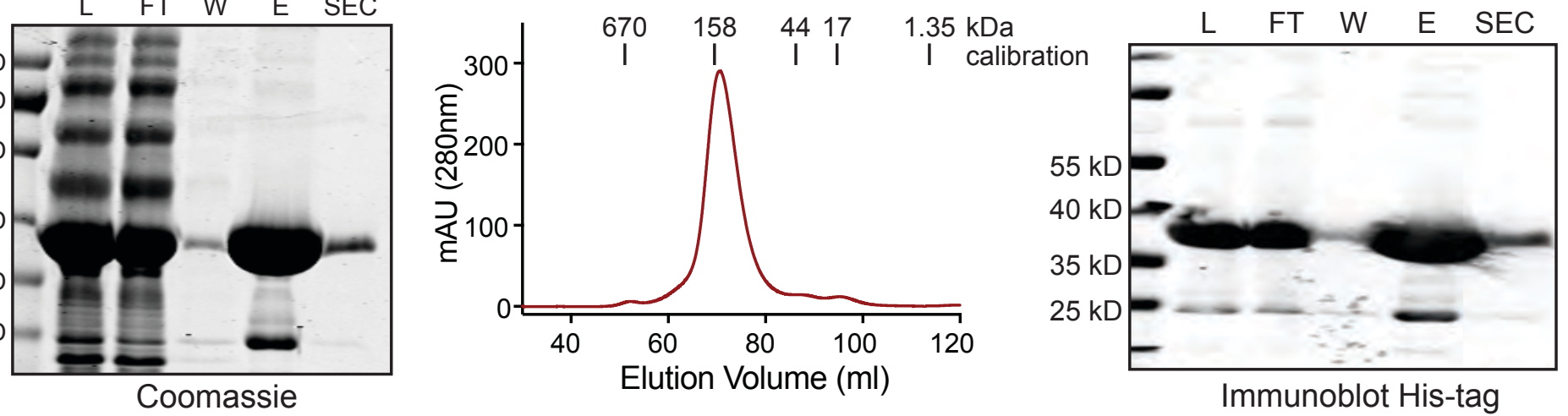

Immunoblot His-tag

30

31

32

33

34

35

36

37

38

39

40

41

42

43

44

45

46

47

48

49

50

51

52

53

54

55

56

57

58

59

60

Figure 1 


\section{Page 27 of 30
a Toxin-emGFP}

ACS Chemical Biology

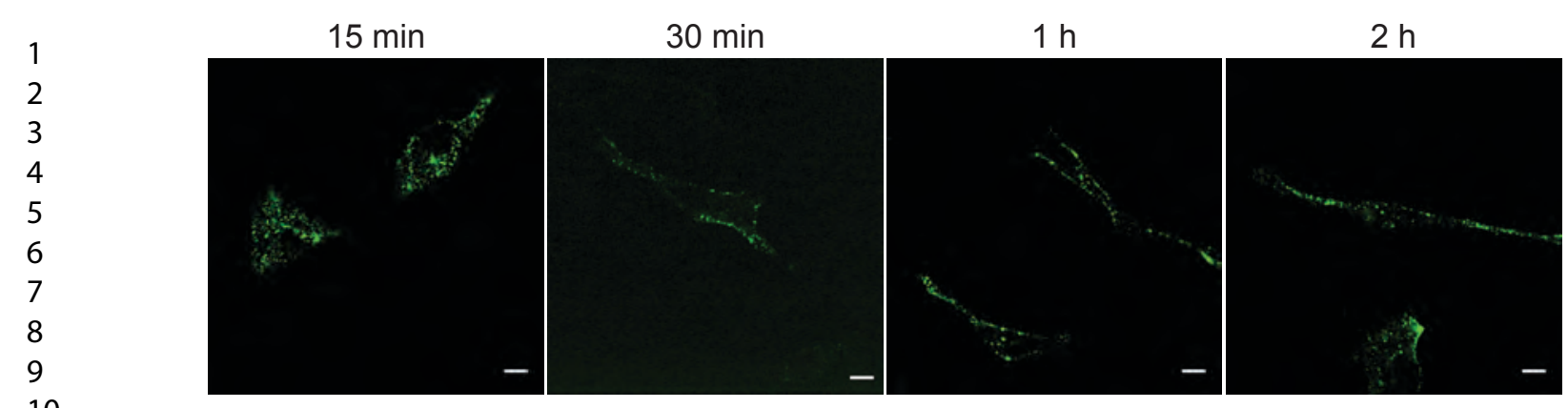

10
11

12

13

14

15

16

17

18

19

$30 \mathrm{~min}$

Toxin-Monobody
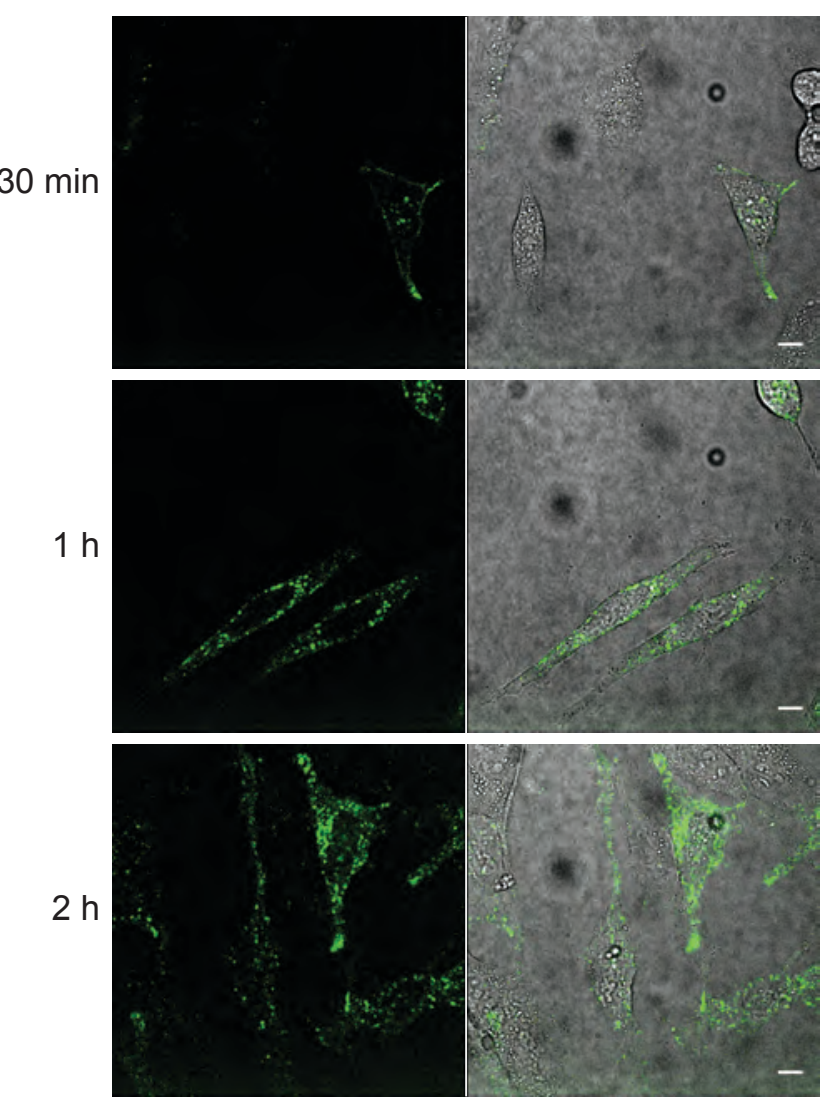

C Toxin-Monobody
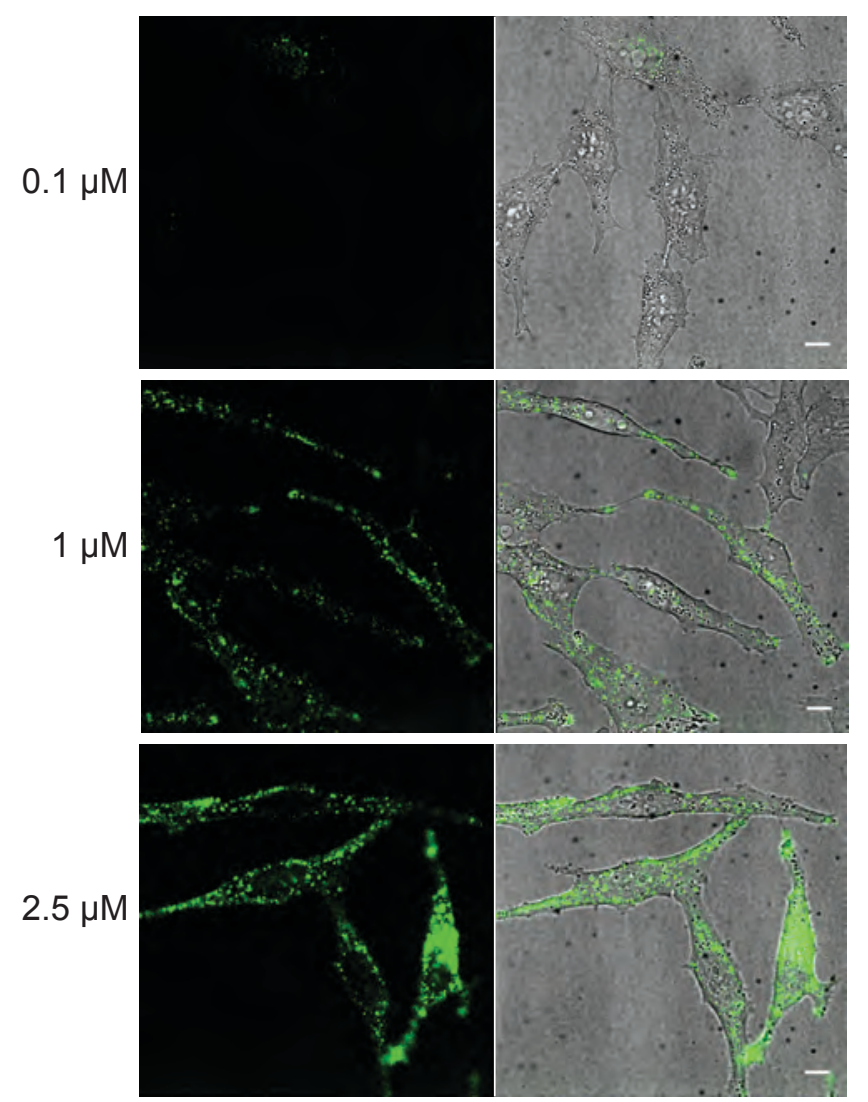

Figure 2 
a Incubation with BG-647-labelled Toxin-SNAP-Monobody
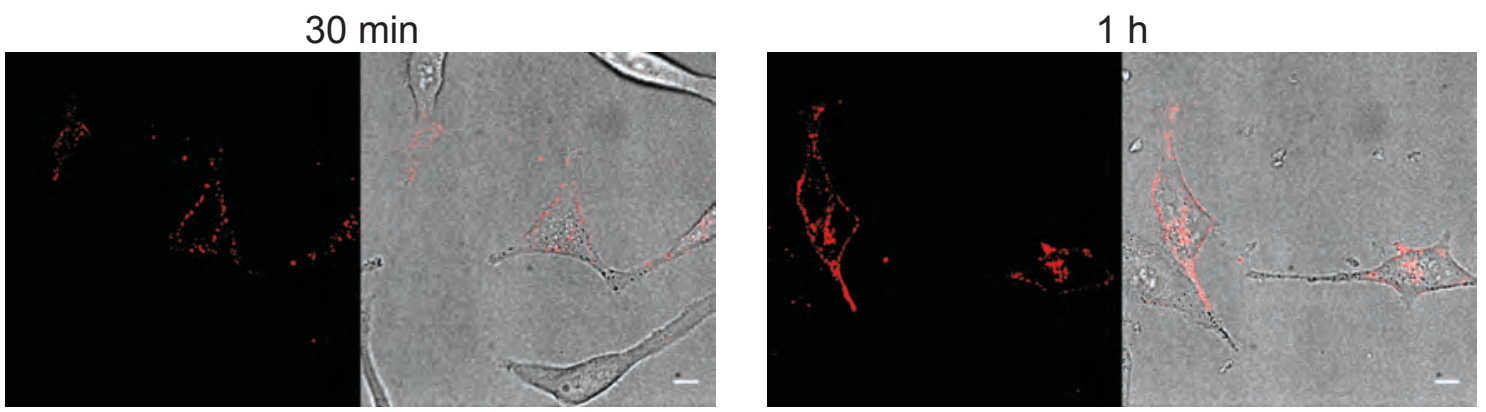

b Incubation with unlabelled Toxin-SNAP-Monobody

C Incubation with BG-647-labelled Toxin-SNAP-Monobody staining with BG-SiR
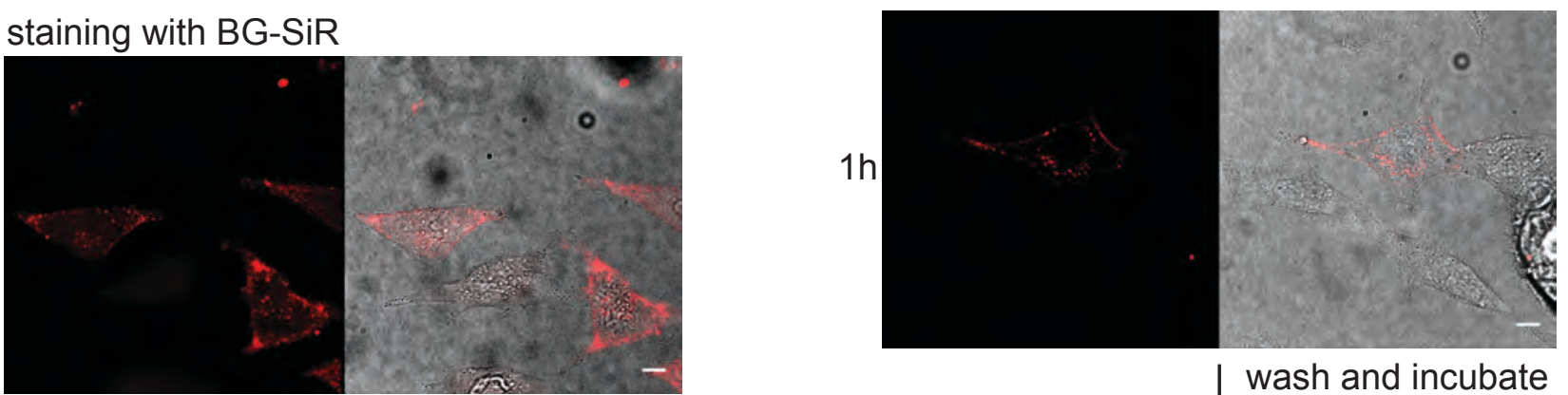

staining with BG-647

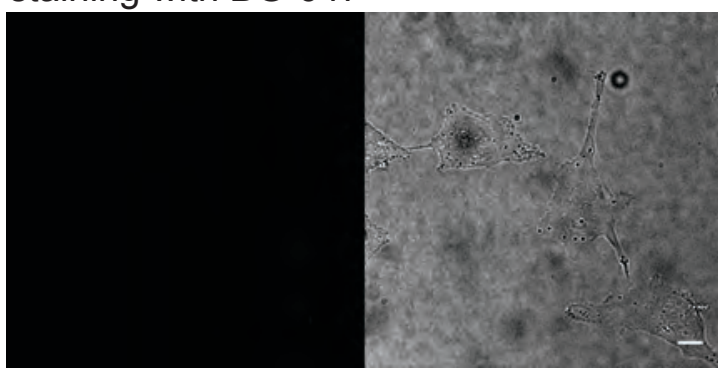

no protein, staining with BG-SiR
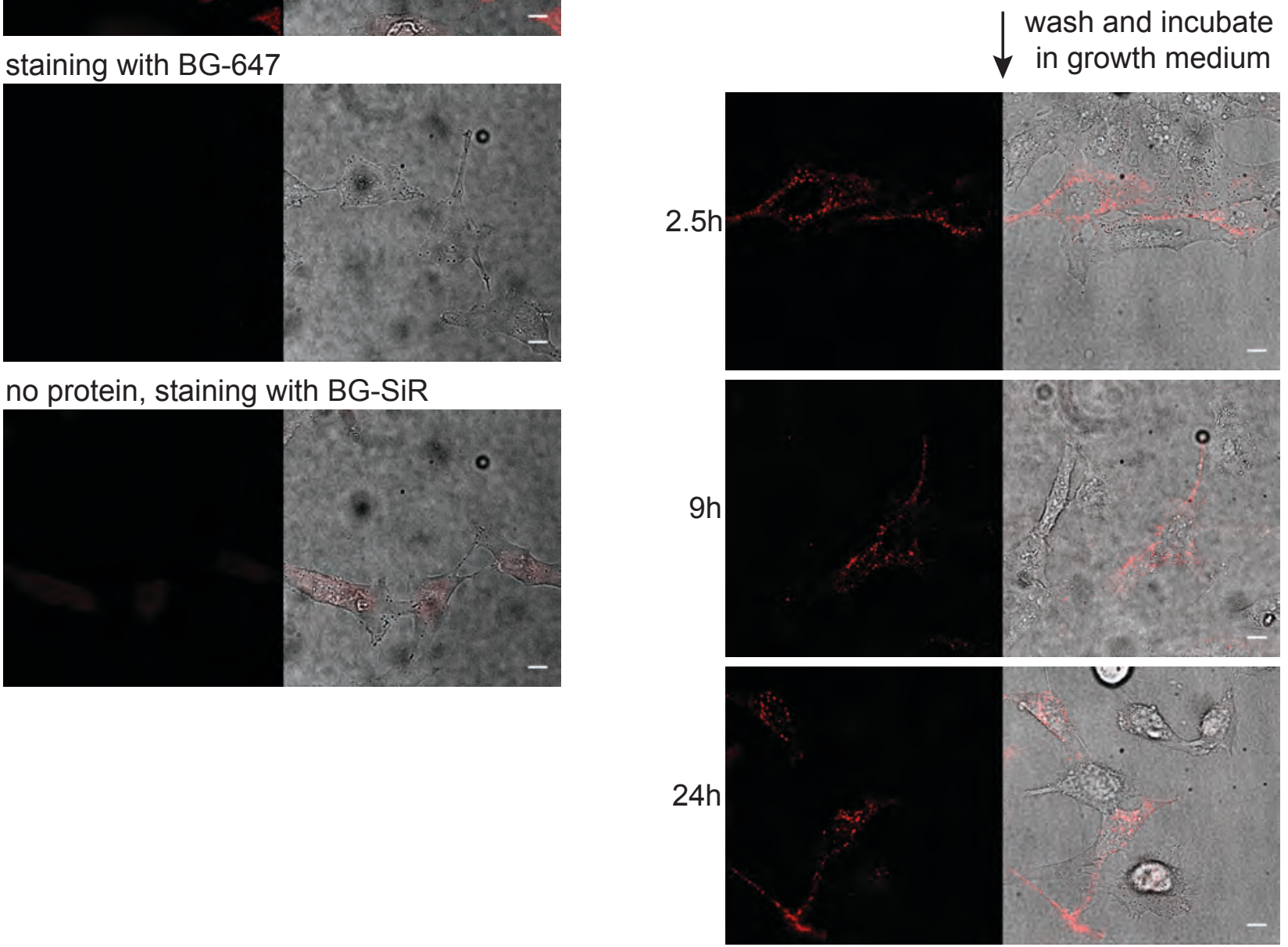

Figure 3 


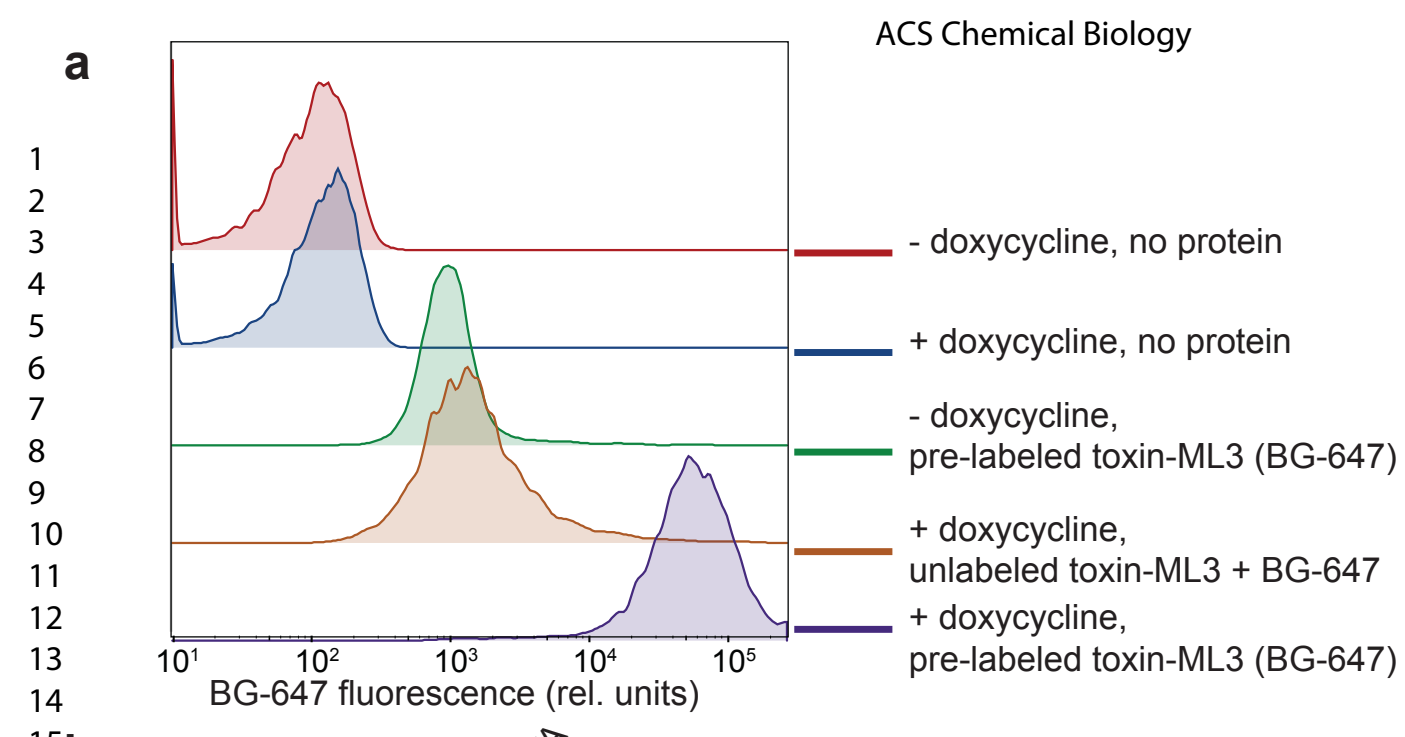

$7 t$

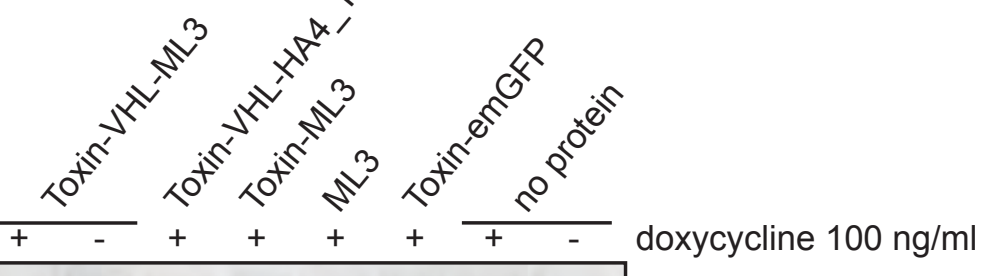

kD-
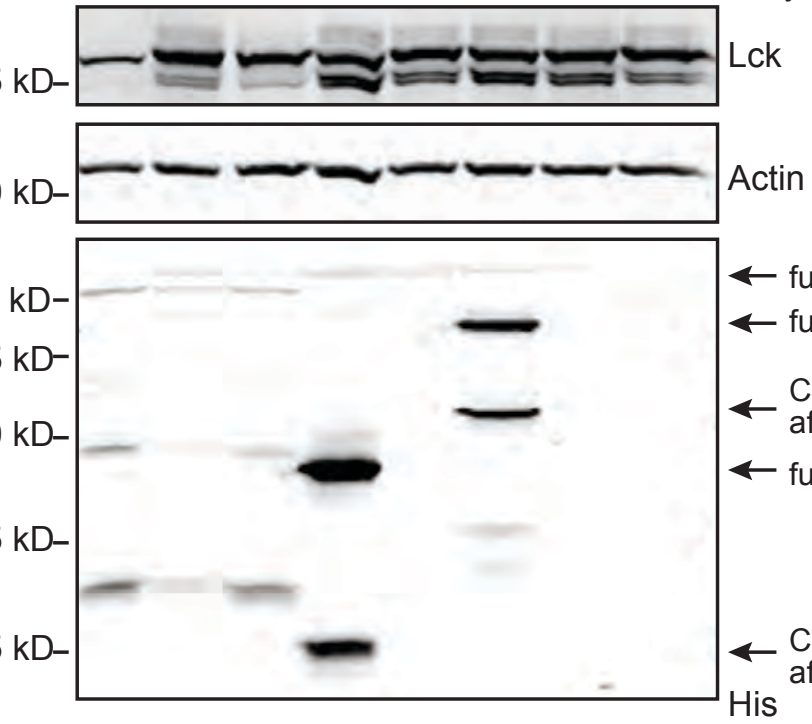

$\leftarrow$ full-length Toxin-VHL-ML3

$\leftarrow$ full-length Toxin-emGFP

C-terminal Toxin-emGFP after furin cleavage

$\leftarrow$ full-length Toxin-ML3

$\leftarrow$ C-terminal Toxin-ML3 His after furin cleavage

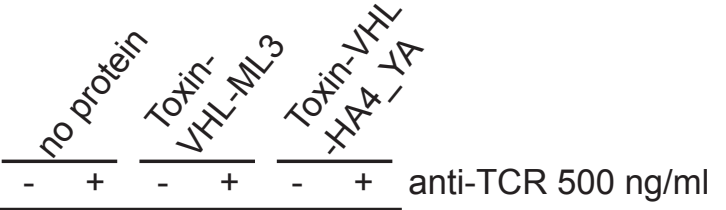

24
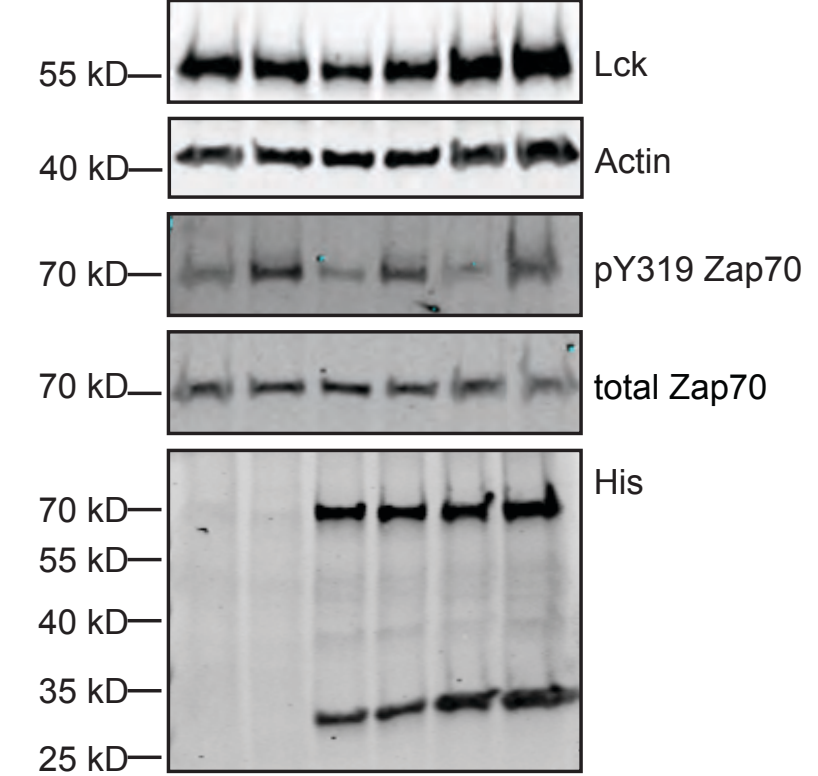

Figure 5
C

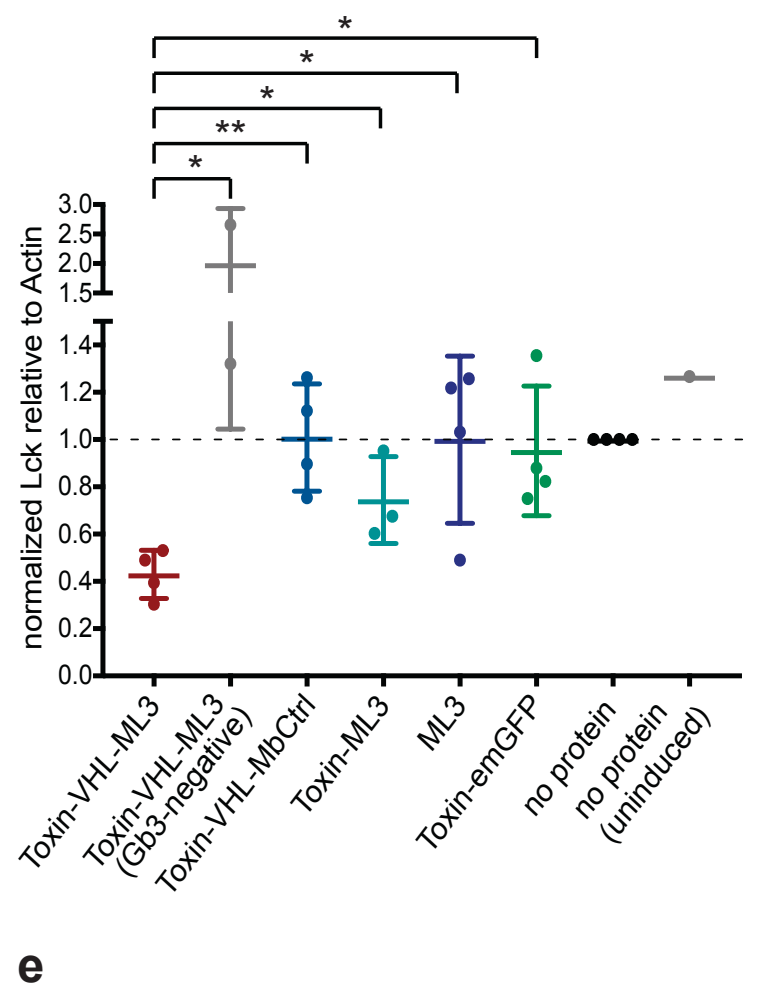

- unstimulated

- anti-TCR $500 \mathrm{ng} / \mathrm{ml}$

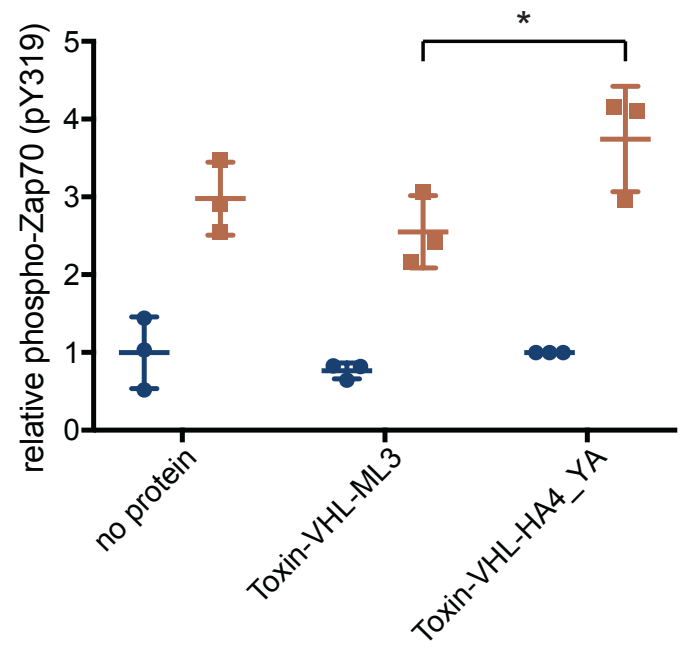

